\title{
Pitavastatin Calcium
}

National Cancer Institute

\section{Source}

National Cancer Institute. Pitavastatin Calcium. NCI Thesaurus. Code C87752.

A calcium salt formulation of pitavastatin, a novel statin that induces plaque regression and elevates HDL-cholesterol levels. 